\title{
Large-Scale Chemical-Genetic Strategy Enables the Design of Antimicrobial Combination Chemotherapy in Mycobacteria
}

\author{
Eachan O. Johnson ${ }^{\dagger}, \$$, , Emma Office ${ }^{\dagger}$, Tomohiko Kawate ${ }^{\dagger, \$,}$, Marek Orzechowski ${ }^{\dagger}$, Deborah T. \\ Hung*, ${ }^{*},+, \$$ \\ ${ }^{\dagger}$ Broad Institute of MIT and Harvard, 415 Main Street, Cambridge, Massachusetts 02142, United \\ States \\ Department of Molecular Biology and Center for Computational and Integrative Biology, \\ Massachusetts General Hospital, 185 Cambridge Street, Boston, Massachusetts 02114, United \\ States \\ ${ }^{\S}$ Department of Genetics, Harvard Medical School, 77 Avenue Louis Pasteur, Boston, \\ Massachusetts 02115, United States \\ *E-mail: hung@broadinstitute.org
}

\section{Supporting information}

\section{Supplemental Figures}

Figure S1 $\mathrm{S}-2$

Figure S2 S-3

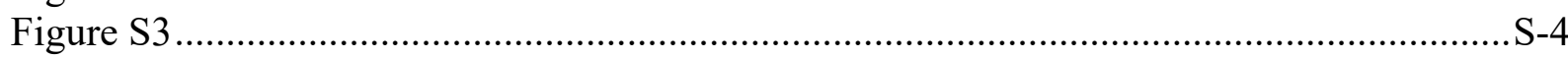




\section{Supplemental Figures}

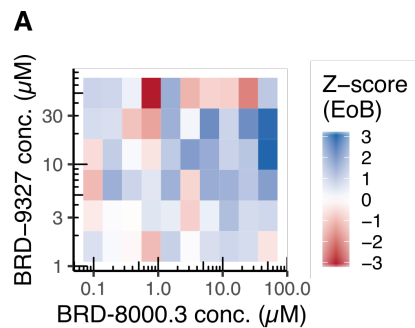

B
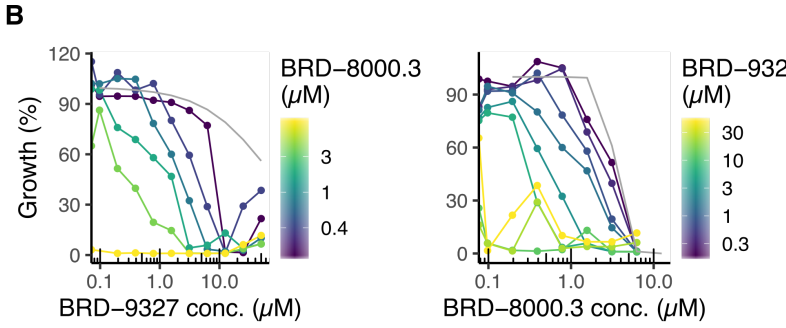

C

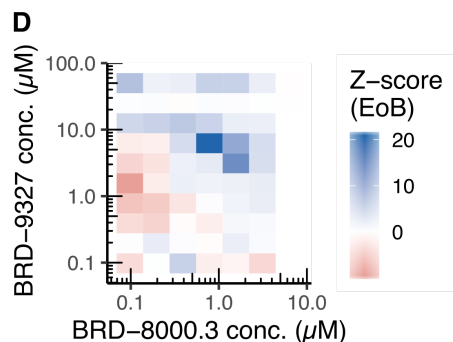

E
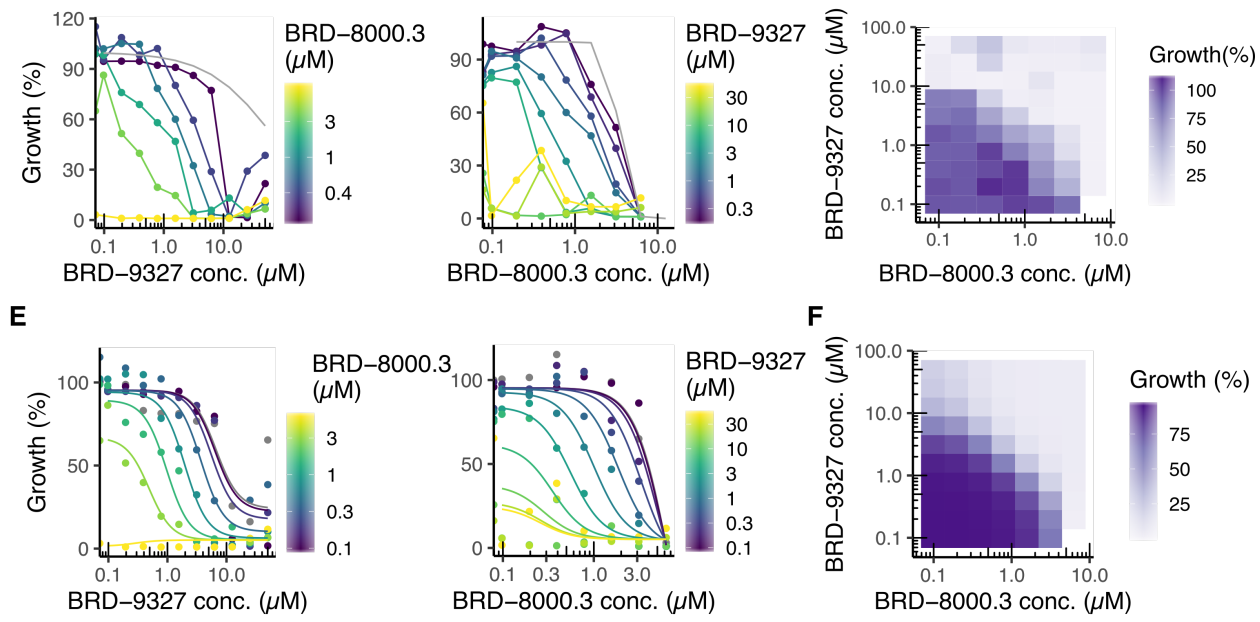

F

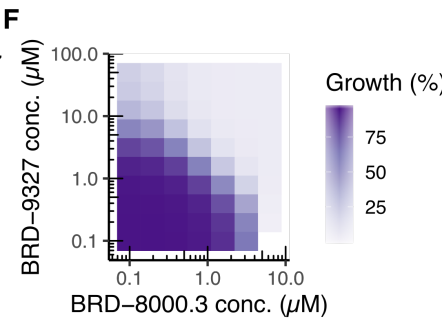

Figure S1. Synergy between EfpA inhibitors BRD-8000.3 and BRD-9327.

(A) Excess-over-Bliss (EoB) of initial EtBr efflux rate inhibition in Msm at varying combined concentrations of BRD-9327 and BRD-8000.3, demonstrating synergy between the two EfpA inhibitors.

(B) Growth inhibition from checkerboard broth microdilution assay of Mmar at varying combined concentrations of BRD-8000.3 and BRD-9327. Filled circles indicate the mean $(n=$ 4).

(C) The data in (B) represented as a heatmap.

(D) Excess-over-Blisss (EoB) calculated for the data in (B), demonstrating synergy between the two EfpA inhibitors. The best fit parameter ( \pm standard deviation) $\alpha=30 \pm 10$ indicates synergy in potency while $\beta=-0.5 \pm 0.1$ indicates modest synergy in efficacy.

(E) Global best fit MuSyC model for the data in (B). Filled circles indicate the mean $(n=4)$.

(F) The best fit MuSyC surface for the data in (B) and (C). 


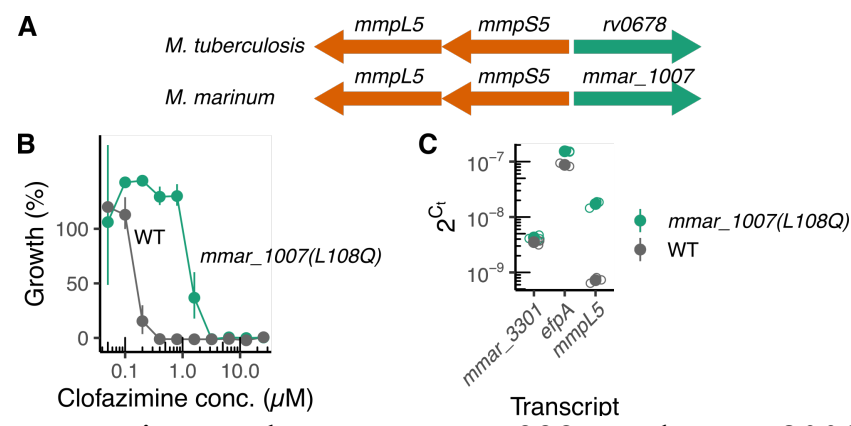

Figure S2. Low-level cross resistance between BRD-9327 and BRD-8000 is mediated by mutation in the efflux pump MmpL5 regulator mmar_1007.

(A) Chromosomal organization of the MmpL5 pump regulator Rv0679 (Mtb) and MMAR_1007 (Mmar), demonstrating synteny.

(B) Broth microdilution assay of wild-type Mmar and Mmar mmar_1007(L108Q) against clofazimine, demonstrating resistance.

(C) Results of a qRT-PCR assay of $m m p L 5$ and efp $A$ transcripts in an mmar_1007 mutant compared to wild-type Mmar, demonstrating 19-fold overexpression of the multidrug efflux pump MmpL5 in mmar_1007 mutants. 

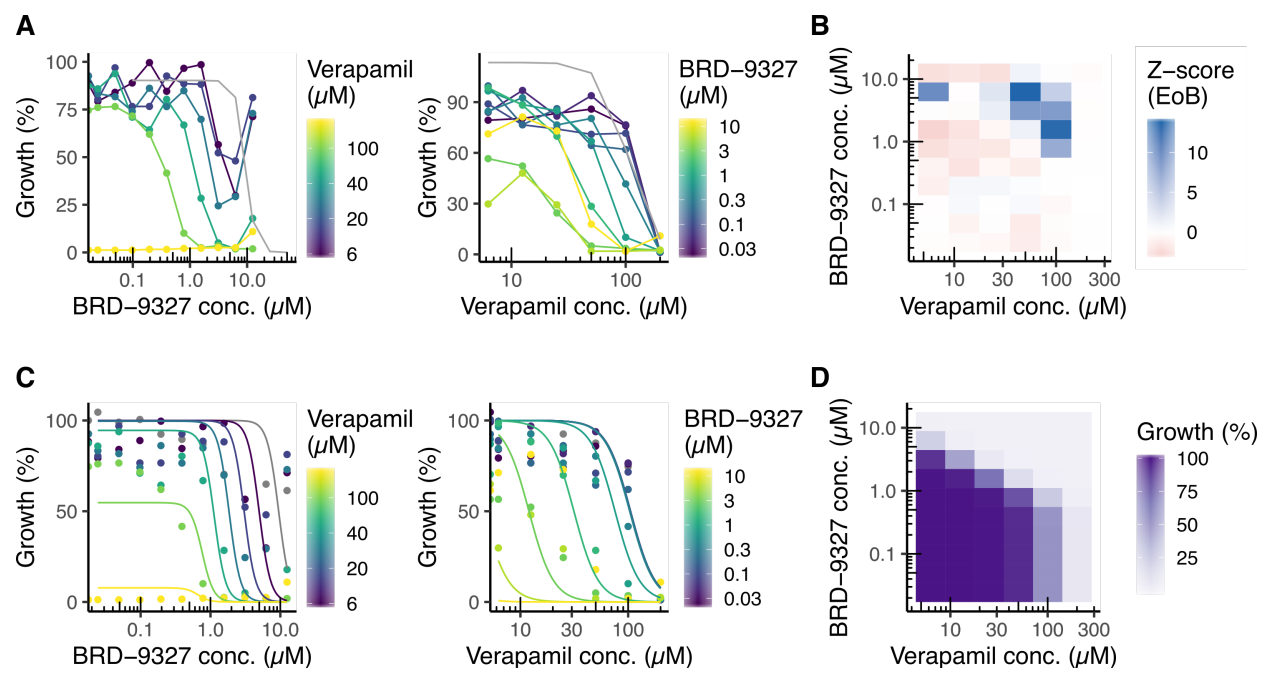

Figure S3. Synergy between BRD-9327 and verapamil.

(A) Growth inhibition from checkerboard broth microdilution assay of Mmar at varying combined concentrations of Verapamil and BRD-9327. Filled circles indicate the mean $(n=4)$. (B) Excess-over-Blisss (EoB) calculated for the data in (A), demonstrating synergy between the two EfpA inhibitors. The best fit parameter ( \pm standard deviation) $\alpha=5 \pm 1$ indicates synergy in potency while $\beta=-0.01 \pm 0.01$ indicates no interaction in efficacy.

(C) Global best fit MuSyC model for the data in (A). Filled circles indicate the mean $(n=4)$. (D) The best fit MuSyC surface for the data in (A). 